\title{
Electronic structure and thermoelectric transport of black phosphorus
}

\author{
L. Craco, ${ }^{1}$ T. A. da Silva Pereira, ${ }^{1}$ and S. Leoni ${ }^{2}$ \\ ${ }^{1}$ Instituto de Física, Universidade Federal de Mato Grosso, 78060-900 Cuiabá, MT, Brazil \\ ${ }^{2}$ School of Chemistry, Cardiff University, Cardiff CF10 3AT, United Kingdom
}

(Received 22 May 2017; published 9 August 2017)

\begin{abstract}
We investigate anisotropic electronic structure and thermal transport properties of bulk black phosphorus (BP). Using density functional dynamical mean-field theory we first derive a correlation-induced electronic reconstruction, showing band-selective Kondoesque physics in this elemental $p$-band material. The resulting correlated picture is expected to shed light onto the temperature and doping dependent evolution of resistivity, Seebeck coefficient, and thermal conductivity, as seen in experiments on bulk single crystal BP. Therein, large anisotropic particle-hole excitations are key to consistently understand thermoelectric transport responses of pure and doped BP.
\end{abstract}

DOI: 10.1103/PhysRevB.96.075118

\section{INTRODUCTION}

Semiconducting black phosphorus (BP) is a layered material [1] with considerable current interest, due to its potential application for optoelectronic [2] and thermoelectric devices $[3,4]$. Recent success in growing two-dimensional, hexagonal "postgraphene" systems [5], including BP monolayers (so-called phosphorene) [6,7], has also intensified the interest in understanding its electronic structure [8,9] and transport properties [10]. BP is the thermodynamic stable allotrope of elemental phosphorus [11], and its layered structure is unique among the elements. Its structure consists of layers of six-membered rings in the chair conformation. A layer can alternatively be viewed as a system of interconnected zigzag chains on two different planes. Within each layer phosphorous atoms are threefold connected. The layer stacking is held together by van der Waals forces to form a three-dimensional crystal as shown in Fig. 1. Due to its intrinsic semiconducting band gap [12-14] and peculiar electronic structure [15] it displays anisotropic particle-hole excitations [16], magnetoresistance [10], and thermal transport properties [4]. Moreover, the band gap of bulk BP is tunable by strain [3] and moderated pressures [17]. Interestingly, electrical resistivity measurements under hydrostatic pressure [17,18] reveal that the one-particle energy gap of orthorhombic BP can be continuously reduced with applied pressure, becoming zero at a critical pressure $P_{c} \approx 1.25 \mathrm{GPa}$ [17]. The low-temperature resistive divergence characteristic of semiconducting systems is gradually suppressed, and beyond $P_{c}$ the resistivity shows (semi)metallic behavior indicating a pressure-induced isostructural insulator-metal transition $[17,19]$. Application of higher pressures generates two consecutive reversible structural transitions: the first one, from an orthorhombic to a rhombohedral phase (gray arsenic type structure) occurring at $5.5 \mathrm{GPa}$ [20], while the subsequent one is from this rhombohedral to a simple-cubic phase at pressures about $11 \mathrm{GPa}$ [21]. Moreover, Hall resistivity measurements of bulk BP close to $P_{c}$ show evidence of topological Lifshitz transition in its band structure. These findings suggest that near the semiconductor-to-semimetal transition, applied pressure pushes the valence and the conduction bands together [17], causing substantial changes in the topology of the Fermi surfaces [19], which is at the origin of the low-energy electronic reconstruction (Lifshitz transition) in pressurized BP.

Despite having been known for over a century now [22], fascinating physics is realized in this puckered layered $p$ band material of rare and intriguing structure. Strain-induced band gap modifications $[3,23,24]$ with tunable semiconductor to anisotropic Dirac semimetal [25] or the existence of a superconducting phase transition under pressure [26] might be linked to selective low-energy electronic reconstruction with characteristics akin to correlated electron systems [27]. Consistent with graphite [28] and elemental bismuth [29], sizable many-body interactions qualifies bulk BP as orbital-selective semimetal hosting renormalized Fermi liquid (FL) electronic states at low energies. Here, we employ dynamical mean-field theory (DMFT) in conjunction with ab initio density functional (DF) calculations [30] to prove the existence of intrinsic many-body correlation effects in the electronic states of bulk BP. Upon carefully comparing the experimentally obtained electronic band dispersions to our theoretical results, we provide further insight about the electronic behavior along the intra- and interlayer directions. Our results are of critical importance in understanding the anisotropic contributions of bulk states near the Fermi level in bulk BP, an aspect of top importance in developing thermal transport devices [4] based on bulk single crystals.

Multiorbital (MO) electronic correlations are intricately tied to the lattice, charge, and spin degrees of freedom [27]. Theoretically, we are still away from being able to treat these coupled correlations in a fully realistic way. Notable progress has been achieved using density functional dynamical meanfield theory (DFDMFT) [30], showing that external perturbations like pressure, electric, or magnetic fields and chemical doping can cause spectacular effects in real materials. Here, we study correlation- and doping-induced electronic reconstructions using the DFDMFT method, which self-consistently takes into account these effects in real materials. An interesting question in the context of correlated semiconductors [31-33] is whether many-body correlation effects, characteristic of narrow band materials [27], can also be hosted in $p$-band semiconducting systems. In this work we study precisely this problem. Based on a realistic modeling, we provide a manyparticle description for the correlated electronic structure and thermal transport of bulk BP. Our results, derived from 


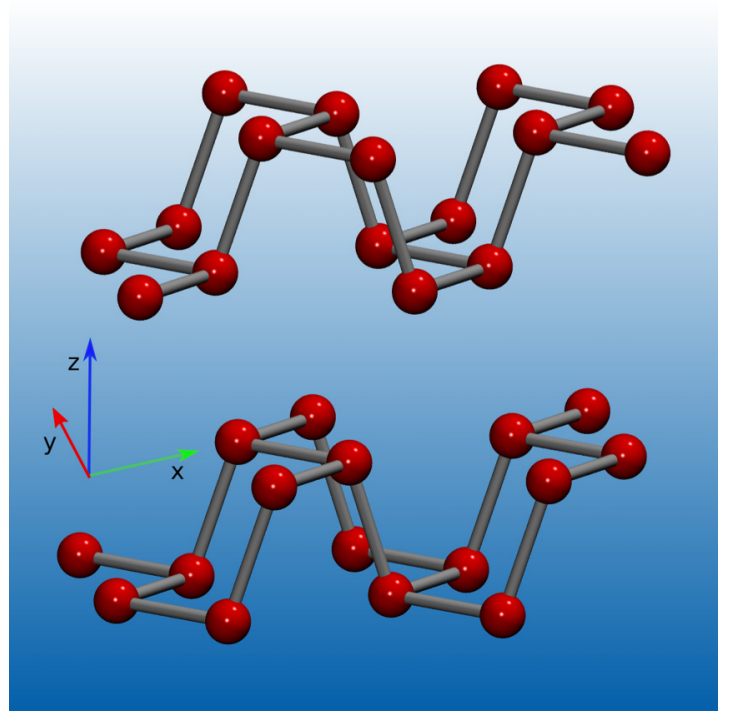

FIG. 1. Layered crystal structure of bulk black phosphorus (BP) with explicit coordinate $(x, y, z)$ axis. Notice the relative positioning of two adjacent puckered sheets consisting of six-connected rings in chair conformation.

reconstructed spectral functions in the bulk, are consistent with polarized, emission, and absorption spectra [15] as well as with resistivity [17] and thermal transport data [4], showing that bulk BP hosts a selective Kondo-like insulating state which is governed by the interplay between an anisotropic band structure and many-body electron-electron interactions.

Kondo insulators are correlated systems [34] whose excitations and normal state properties are adiabatically connected to noninteracting semiconductors [35]. Hence they can be seen as an analytically continued version of conventional band insulators, where sizable correlations exist above the one-particle gap scale. In Kondo insulators, when the band filling is slightly different from its commensurate value, a coherent electronic cloud is created close to $E_{F}$ and the resulting metallic state (in absence of disorder or proximity to quantum criticality) is Fermi liquid (FL). With this in mind, in the present work we clarify why MO electronic correlations are the clue to an anisotropic electronic state in bulk BP at ambient conditions. Moreover, we reveal the existence of a first-order semiconducting-to-metal transition on increasing on-site Coulomb interaction $U$ in this $p$-band fermion system.

\section{RESULTS AND DISCUSSION}

In bulk BP individual layers are weakly connected by van der Waals forces, as opposed to strong intralayer covalent bonds. BP has an orthorhombic unit cell with space group Cmca (No. 64) [3]; lattice parameters $a=4.38 \AA$, $b=3.31 \AA$, and $c=10.5 \AA$ [36]. As shown in Fig. 1, each individual BP layer consists of two parallel planes, each containing zigzag chains of phosphorous atoms extending along the $y$ direction [15]. Therein, each phosphorus atom has three nearest neighbors, two on the same zigzag chain and the third one located on a different zigzag chain on the other plane. Similar to graphite [28] and elemental bismuth [29], we performed first-principle local density approximation (LDA)

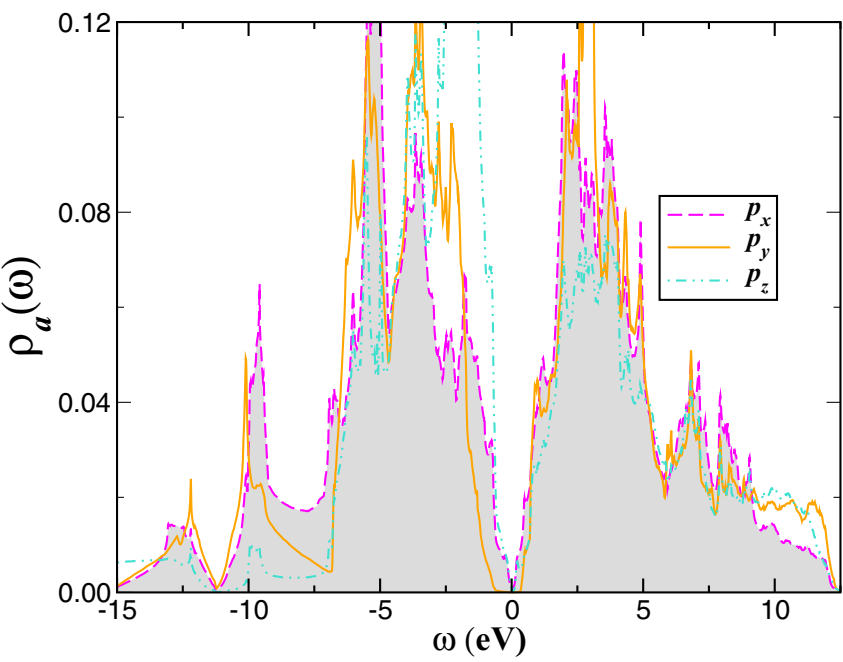

FIG. 2. LDA orbital resolved and density of states (DOS) of bulk $\mathrm{BP}$, showing a pronounced semiconducting band gap in the $p_{y}$ channel at low energies. Notice the broad one-particle bandwidth, $W \approx 27 \mathrm{eV}$, characteristic of bulk $p$ band systems.

calculations with the PY-LMTO package [37]. Experimental crystal structure parameters were used [36]. The total density was converged on a grid of 301 irreducible $k$ points, and the radii of the atomic spheres were chosen as $r=2.74$ a.u. in order to minimize their overlap. Consistent with previous calculations for bulk BP [15] our LDA results in Fig. 2 confirm that the active electronic states in this system involve the $3 p$ carriers, where all three $p$ bands have appreciable weight below and above the Fermi energy, $E_{F}(=\omega=0)$. Also consistent with previous calculations [13], our results reveal an anisotropic one-particle band structure.

\section{A. Correlated electronic structure of bulk BP}

MO dynamical electron-electron interactions often drive spectacular effects in real materials [27]. Precisely how this might come about is an open, challenging problem also for wide band systems. Here, we study correlation induced one-particle reconstruction in BP using combined LDA and DMFT methods [30]. In recent years, this scheme was used to shed light onto transport anisotropy in graphite [28] and on the electronic structure of elemental bismuth [29], which is the heavier group homologue, and which also display a layered, gray arsenic type structure. Both studies show good agreement with spectroscopy (graphite) and electrical transport (bismuth) measurements. In this work, we use LDA+DMFT to study the electronic structure and thermal transport properties of bulk BP. As a by-product we reveal an interaction-induced electronic reconstruction in bulk BP at low energies which is relevant to thermoelectricity of correlated narrow band semiconductors.

The many-body MO Hamiltonian relevant for bulk BP is $H=H_{0}+H_{\text {int }}$ with $H_{0}=\sum_{\mathbf{k} a \sigma} \epsilon_{a}(\mathbf{k}) c_{\mathbf{k} a \sigma}^{\dagger} c_{\mathbf{k} a \sigma}$ and

$$
H_{\text {int }}=U \sum_{i a} n_{i a \uparrow} n_{i a \downarrow}+\sum_{i a \neq b} U^{\prime} n_{i a} n_{i b}-J_{H} \sum_{i a \neq b} \mathbf{S}_{i a} \cdot \mathbf{S}_{i b} .
$$



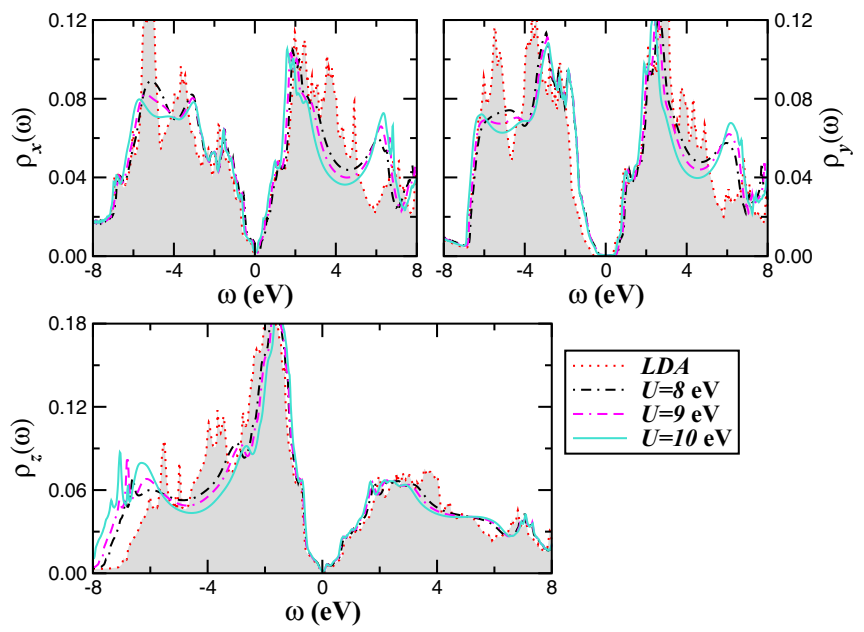

FIG. 3. LDA and LDA+DMFT orbital-resolved density of states (DOS) of bulk BP. An important feature of the LDA results is the fact that all $3 p$ bands span over the Fermi level, $E_{F}=\omega=0$. Notice as well the anisotropic band gap in LDA and the transfer of spectral weight induced by many-body electron-electron interactions within DMFT. Noteworthy are the crossing points in the LDA+DMFT spectral functions, which are fingerprints of many-body correlation effects.

Here, $a=x, y, z$ label the diagonalized $3 p$ bands and $\epsilon_{a}(\mathbf{k})$ is the one-electron band dispersion, which encodes details of the one-electron (LDA) band structure. $U^{\prime} \equiv U-2 J_{H}$, with $U, U^{\prime}$ being the intra- and interorbital Coulomb repulsion and $J_{H}$ being Hund's rule coupling. Within LDA the one-band dispersions are read off from $\epsilon_{a}(\mathbf{k})$ : these are inputs for MO LDA+DMFT, which generates a Kondo insulating state in bulk BP as shown below. We use MO DMFT for the threeorbital model of bulk BP with the MO iterated perturbation theory (MO-IPT) as impurity solver. The detailed formulation of MO-IPT for correlated electron systems has already been described in detail [38] and used in the context of elemental $p$-band semimetals in Refs. [28,29], so we do not repeat the equations here.

To pinpoint the excitation spectrum that emerges from dynamical MO electron-electron interactions in bulk BP, we present in Fig. 3 our LDA+DMFT results for $8 \leqslant U \leqslant 10 \mathrm{eV}$ with fixed $J_{H}=0.5 \mathrm{eV}$. [Our choice for $U$ is consistent with Hartree-Fock estimation of intra-atomic Coulomb energy [39] as well as with the bare (unscreened) on-site Coulomb correlation parameter recently predicted for the $p_{z}$ states of single-layer BP [40] and puts bulk BP on the Kondo insulating side of the correlated semiconducting family [31,32].] The emergence of a narrower (compared to LDA) insulating gap at low energies with concomitant appearance of lower(LHB) and upper-Hubbard (UHB) bands on different orbitals at high energies with increasing $U$ is visible in Fig. 3. As is common to a system showing screened many-body effects, electron-electron interactions partially modify the bare, LDA spectral functions of bulk BP. In spite of the small $U / W \approx 0.5$ parameter ratio used here ( $W$ is the one-particle LDA bandwidth), MO dynamical correlations arising from $U, U^{\prime}$ lead to spectral weight redistribution over large energy scales. Noticeable differences in the spectral weight transfer
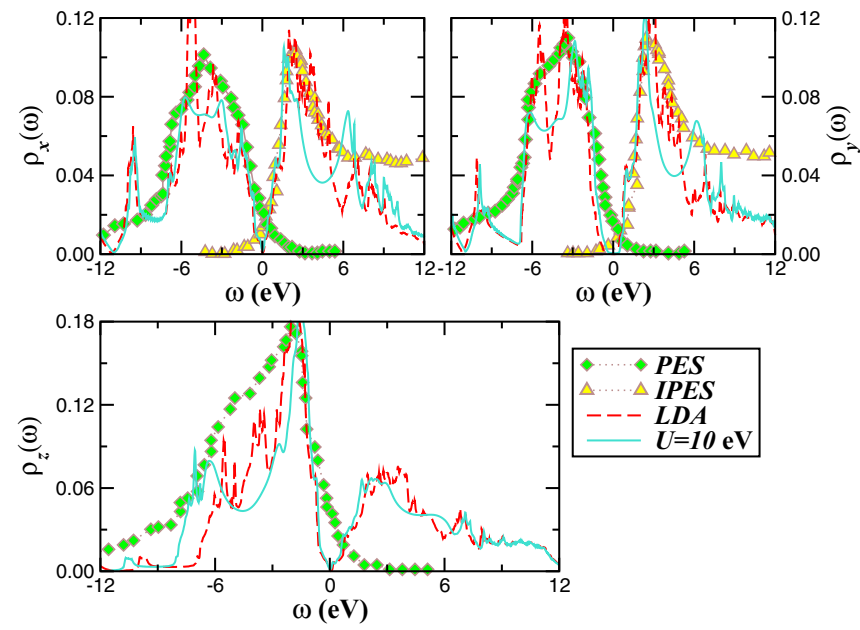

FIG. 4. Comparison between the LDA and LDA+DMFT $(U=10 \mathrm{eV})$ result for bulk BP (dashed and solid lines, respectively) and angle-integrated photoemission (PES, diamonds) and inverse photoemission spectroscopy ( $K$ absorption, triangles) taken from Hayasi et al. [15]. Good quantitative theory-experiment agreement at energies between $-6 \leqslant \omega \leqslant 6 \mathrm{eV}$ is visible. In particular, the low-energy line shapes on different orbital channels are accurately resolved. Notice the substantial improvement obtained by LDA+DMFT over the LDA result for $p_{z}$-bump feature in PES close to $6.5 \mathrm{eV}$ binding energy.

(SWT) are seen between the $p_{z}$ and $p_{x, y}$ channels. Within the $p_{z}$ orbital the LHB at $\omega \approx 6.0 \mathrm{eV}$ for $U=8 \mathrm{eV}$ (and $U^{\prime}=7 \mathrm{eV}$ ) is clearly resolved and moves to higher energies with increasing $U$. SWT is also seen within the planar orbitals. Interestingly, in these orbitals the UHBs close to $6 \mathrm{eV}$ in the conduction band are more clearly resolved compared to the $p_{z}$ orbital. This can be taken as a fingerprint of asymmetric, orbital-selective Coulomb correlation effects in bulk BP which might be relevant to understanding anisotropic particle-hole excitations probed in optics [16]. While large transfer of spectral weight from lower to higher energies is obtained, the spectral line shape close to $E_{F}$ remains close to that found in LDA. As visible in Fig. 3, only the $p_{y}$ channel shows partial reduction of the one-particle band gap due to many-body correlation effects.

Since the dependence of electron-electron interactions in the excitation spectrum of postgraphenelike systems is quite subtle and not yet fully understood, in Fig. 4 we compare our orbital-resolved LDA and LDA+DMFT $(U=10 \mathrm{eV})$ spectral functions with polarized photoemission (PES) and inverse-photoemission (IPES) spectroscopy data for bulk BP [15]. Good semiquantitative agreement with both PES and IPES results is obtained at low energies. In particular, the low-energy line shapes are faithfully reproduced. Remarkably, our LDA+DMFT calculation reproduces the large SWT of the $p_{z}$ orbital at high binding energies, without convoluting the theoretical curve as in Ref. [15]. Here, the bump feature seen in PES close to $-6.5 \mathrm{eV}$ are interpreted as arising from collective intra- and interorbital local-moment fluctuations within the LHB of the $p_{z}$ orbital state. Moreover, compared to LDA the charge carriers of BP are in a Fermi liquid (FL) regime, characterized by the emergence of narrow Kondo 

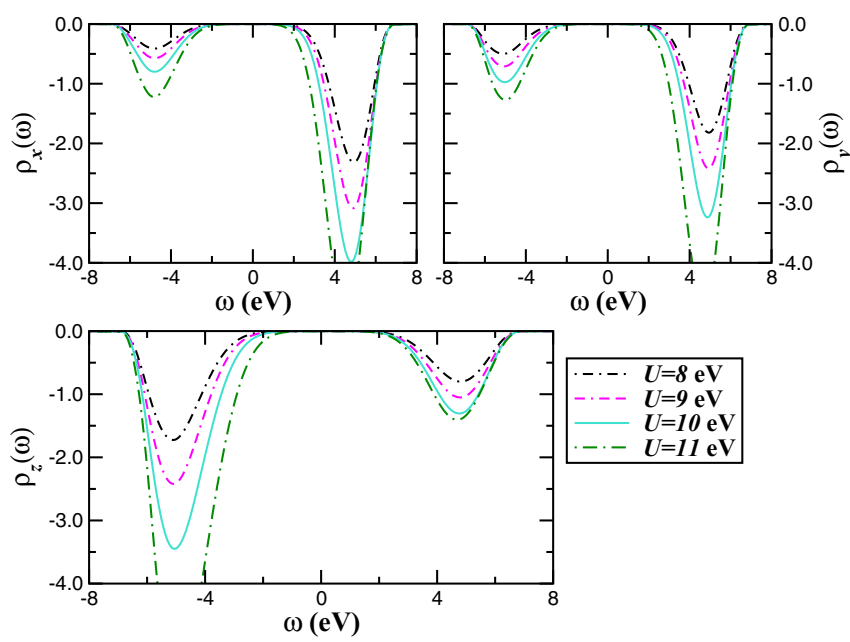

FIG. 5. Imaginary part of the orbital-resolved self-energies $\left[\operatorname{Im} \Sigma_{a}(\omega)\right]$ within the Kondo insulating phase bulk BP for different values of $U$ and $J_{H}=0.5 \mathrm{eV}$.

quasiparticle resonances in the orbital-selective DOS near $E_{F}$. This behavior is often seen in Kondo insulators, where local quantum fluctuations induce the FL low-energy coherence. Thus, in bulk BP, the transfer of spectral weight found in the FL semiconducting phase can be traced to a dynamical scattering process that leads to electron mass enhancement consistent with experimental observations [16]. Taken together, our results constitute a consistent, quantitative rationalization of basic one-particle responses of bulk BP and serve as a starting point to explore the orbital reconstruction upon electron and hole doping the parent compound; see our study below.

To further illustrate the correlated nature of our spectral functions near the gap energy scale, in Fig. 5 we show the changes in the orbital-resolved imaginary parts of the selfenergies $\left[\operatorname{Im} \Sigma_{a}(\omega)\right]$. Looking closely at these self-energies a remarkable aspect stands out: $\operatorname{Im} \Sigma_{a}(\omega)$ vanishes in the region of the semiconducting gap, instead of having a pole, as would occur in a strongly correlated Mott insulator [27]. This aspect is reminiscent of a Kondo insulator, where the gap arises due to sizable interband hybridization [38]. The semiconducting behavior of bulk BP is, in our approach, that of a correlated Kondo insulator. As discussed earlier [13], within the puckered lattice structure of BP a narrow semiconducting band gap opens up because of the orbital-dependent $p$-band hybridization. Additionally, as shown here, correlation effects in conjunction with band structure details play a crucial role. As is apparent from the orbital-resolved self-energies, sizable correlation effects manifest themselves above the gap scale as negative bump structures, unveiling underlying Kondesque as in the graphite system [28]. While this behavior is somewhat unexpected for bulk BP, proximity to an anisotropic Kondo insulating state is seen by observing the pronounced negative bump structures in $\operatorname{Im} \Sigma_{a}(\omega)$ lying above $(x, y)$ and below $(z)$ the Fermi energy $E_{F}$. Since the system is intrinsically correlated, spectral redistribution in response to additional small perturbations (e.g., external pressure or chemical doping) can drive it into a true metallic state which in turn might host superconductivity [19] at low temperatures. More importantly (see below) electron and hole doping will also cause largescale orbital-selective spectral weight transfer and metallicity in bulk BP.

\section{B. Electrical and thermal transport properties of pure and doped $\mathrm{BP}$}

The Seebeck coefficient, like the electrical and thermal conductivities, is exactly computable within DMFT [41] using the fully renormalized DMFT propagators. Here, we determine the transport coefficients using the generalized Kubo formulation to the three-band case relevant for bulk BP [42]. The general expressions for Seebeck $S$ and thermal conductivity $k$, which, respectively, measures the mixed electrical-thermal correlations $[S(T)]$ and the heat-current correlations $[k(T)]$ at finite temperatures are

$$
\begin{gathered}
S(T)=\frac{1}{T} \frac{A_{1}(T)}{A_{0}(T)}, \\
k(T)=\frac{1}{T}\left(A_{2}(T)-\frac{A_{1}^{2}(T)}{A_{0}(T)}\right),
\end{gathered}
$$

with

$$
A_{n}(T)=\frac{e^{2}}{e^{n} \hbar} \int_{-\infty}^{+\infty} d \omega \phi(\omega)\left[-f^{\prime}(\omega)\right](\omega-\mu)^{n}
$$

and

$$
\phi(\omega)=\frac{1}{V} \sum_{\mathbf{k}} \operatorname{Tr}\left[v_{a}(\mathbf{k}) \rho_{a}(\mathbf{k}, \omega) v_{b}(\mathbf{k}) \rho_{b}(\mathbf{k}, \omega)\right] .
$$

Here,

$$
\rho_{a}(\mathbf{k}, \omega)=-\frac{1}{\pi} \operatorname{Im}\left[\frac{1}{\omega+\mu-\Sigma_{a}(\omega)-\epsilon_{\mathbf{k}, a}}\right]
$$

is the LDA+DMFT spectral function, $a=(x, y, z)$ represents the three $p$ orbitals, $\mu$ is the chemical potential, $v_{a}(\mathbf{k})$ is the group velocity, $f(\omega)$ is the Fermi function, and $V$ the sample volume. In LDA+DMFT, the $A_{n}(T)$ are convertible to integrals over the unperturbed LDA DOS. As in Refs. [29,42], the only approximation made here is to ignore the $\mathbf{k}$ dependence of $v_{a}(\mathbf{k})$. A compelling reason for justifying this approximation is that different sources of scattering, neglected in our LDA+DMFT formulation but present in reality, like phonons and lattice defects, will partially degrade the quasiparticle momentum. In this situation, following Saso et al. [43], we approximate $v_{a}(\mathbf{k})$ by a single average carrier velocity $v$ for all orbitals. In fact, Saso et al. [43] and Baldassare et al. [44] have shown that this assumption works well for Kondo insulators $\left(\mathrm{FeSi}\right.$ and $\left.\mathrm{YbB}_{12}\right)$ as well as for $\mathrm{V}_{2} \mathrm{O}_{3}$, supporting our approximation in Eq. (5). The observed features in the dc resistivity $\rho_{d c}(T)$, thermal conductivity $k(T)$, and thermoelectric power $S(T)$ originate from correlation and doping induced spectral changes: here, our focus is to show that this provides a compelling description of extant experimental data.

We now describe our transport results. In Fig. 6 we display the $T$ dependence of electrical resistivity $\left[\rho_{d c}(T) \equiv 1 / A_{0}(T]\right)$ with increasing $U$, computed using the LDA+DMFT orbital resolved spectral functions in Eq. (5) above. Various interesting features immediately stand out. First, $\rho_{d c}(T \rightarrow 0)$ is large 


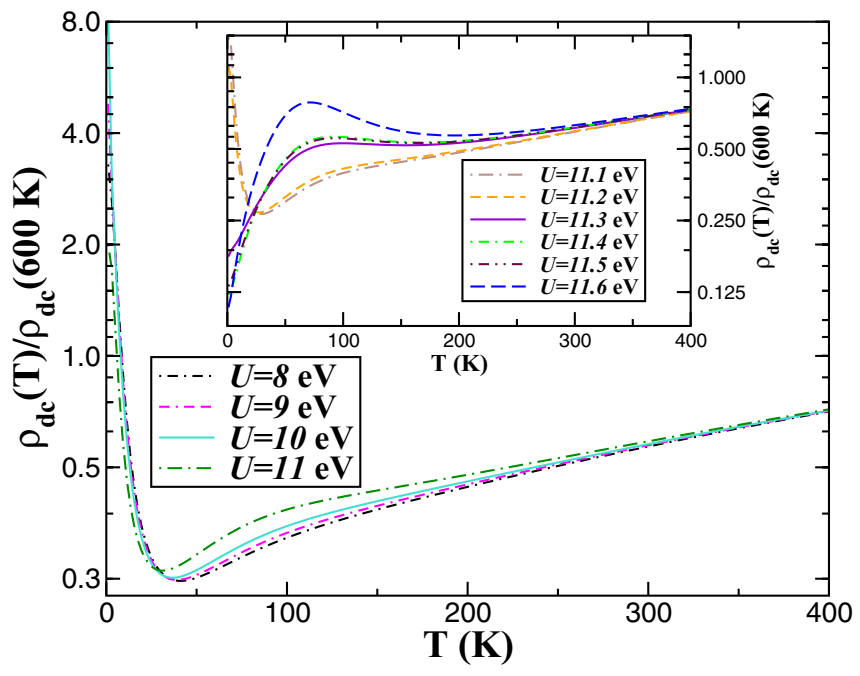

FIG. 6. $T$ dependence of electrical resistivities for the Kondo insulating state of bulk BP, showing qualitative good accord with transport data [10,17]. Particularly interesting is the insulating behavior at low temperatures intrinsic to narrow band semiconductors and the minimum value around $40 \mathrm{~K}$. Inset display resistivity curves at higher $U(>11.0 \mathrm{eV})$, showing a correlation-induced first order semiconducting-to-metal transition at a critical Coulomb interaction.

with clear semiconductinglike behavior at low temperatures, in accordance with the insulating description above. Similar $T$ dependence as in Fig. 6 for $U=10 \mathrm{eV}$ has been observed in Ref. [10], where the exponential increase at low- $T$ is governed by an intrinsically small band gap energy $\left(E_{g}=\right.$ $14.6 \mathrm{meV}$ ) in contrast to previous reports [12]. Also consistent with experimental observations [10] is the fact that with decreasing temperature from high- $T(\approx 250 \mathrm{~K}$ in experiment $)$ $\rho_{d c}$ decreases in a metallic form reaching a minimum value around $40 \mathrm{~K}$. While in experiment this value is close to $60 \mathrm{~K}$, the good qualitative agreement between theory and experiment can serve as a proof of the narrow band semiconducting nature of bulk BP. However, this localized behavior is smoothly suppressed with increasing $U$ and disappears in the correlation-induced metallic phase at $U_{c}=11.3 \mathrm{eV}$; see inset of Fig. 6. Noteworthy is that above this critical value the resistivity curves show a small insulating upturn below $200 \mathrm{~K}$ accompanied by normal state metallicity from $70 \mathrm{~K}$ down to zero temperature. Interestingly, the $T$ dependence in the inset of Fig. 6 resembles the one seen in experiments of pressurized BP [17], showing pressure-induced insulator-metal transition with increasing pressure from ambient pressure to $2.4 \mathrm{GPa}$. Based on our results it is plausible to assume that noticeable electronic reconstruction occurs in pressurized BP. These results strengthen the view that bulk BP is a correlated semiconductor proximate to a Kondo metal [45].

Expanding on a previous work [42], in what follows we provide a microscopic description of anisotropic insulating and metallic phases of doped BP in depth. Specifically, in addition to our indications from dc resistivity, we show how the Seebeck coefficient and thermal conductivity (electronic part) are also described, and correlate with evolution of the LDA+DMFT spectral functions with doping, $\delta$. We correlate the anisotropic evidences from different transport studies $[3,4]$
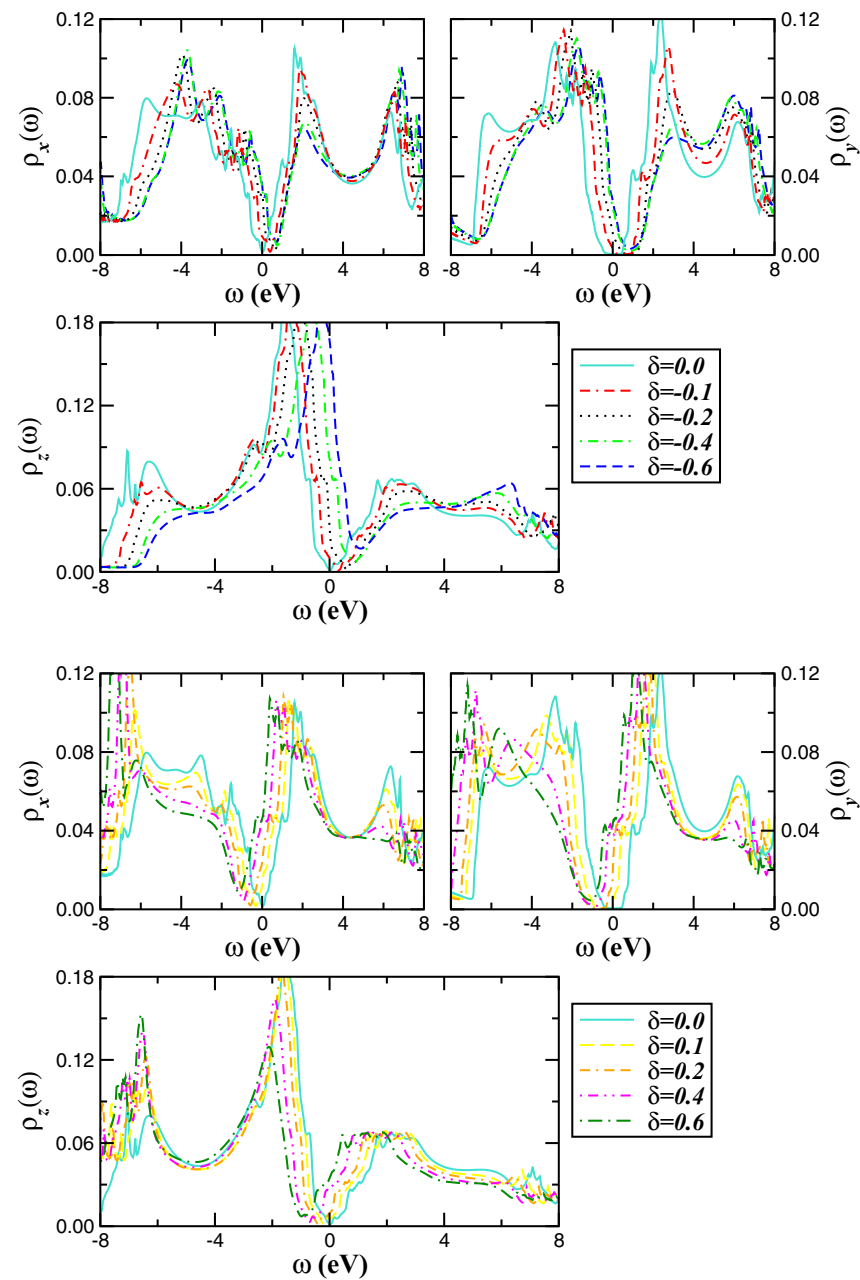

FIG. 7. Orbital resolved LDA+DMFT $\left(U=10.0 \mathrm{eV}, J_{H}=\right.$ $0.5 \mathrm{eV}$ ) spectral functions for the semiconducting and metallic phases of bulk BP, showing their evolution with increasing electron (lower panels) and hole (upper panels) doping, $\delta$. The energy gap suppression with increasing $\delta$ goes hand-in-hand with large scale spectral weight transfer. Particularly interesting are the isosbestic (crossing) points at different energies in the spectral functions of doped BP.

in a single correlation-based scenario. This reinforces the basic hypothesis about the role of MO many-body correlations in this bulk $p$ band semiconductor.

Given the large dynamical SWT characteristic of correlated electron systems, electron/hole doping can be expected to have a sensible impact on a correlated semiconductor [32] close to an insulator-to-metal instability. Even though data exists only for pressurized BP [17], the generic appearance of novel metallic states and the instabilities of such states to, for example, hole-carrier superconductivity [19] makes this a very important question worth investigating.

In Fig. 7 we show the changes in the correlated electronic structure upon electron/hole doping $(\delta \neq 0)$ the parent compound. An intriguing observation in Fig. 7 is that the localization-delocalization transition does not occur simultaneously in all orbitals at small doping levels. As $\delta$ increases to negative or positive values, a selective-metallic state develops, in which the $p_{y}$-orbital spectral functions show 

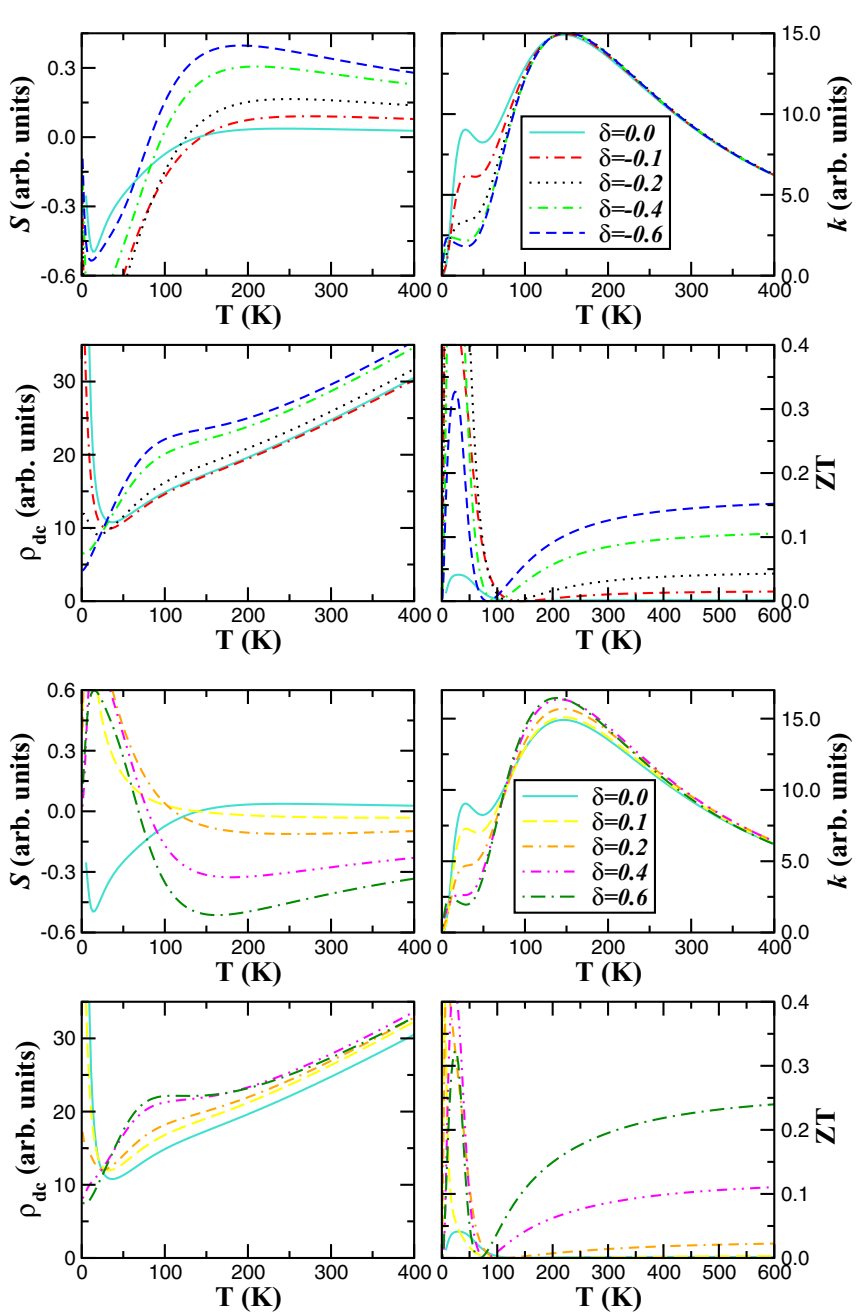

FIG. 8. Seebeck coefficient $(S)$, thermal conductivity (electronic part) $(k)$, resistivity $\left(\rho_{d c}\right)$, and the figure of merit $(Z T)$ for pure, electron- (bottom panels), and hole- (upper panels) doped BP obtained within LDA+DMFT with $U=10 \mathrm{eV}$. Notice the change in sign in $S(T)$ around $100 \mathrm{~K}$ upon doping the parent compound. Also interesting is the $T$ dependence of the thermoelectric figure of merit, $Z T$, showing high values at low temperatures. Intriguing similarities with thermal conductivity data by Wang et al. [4] are visible.

semiconductinglike behavior with vanishing DOS at $E_{F}$, while the $p_{x, z}$ orbitals show (selective) metallic behavior, characterized by the presence of low-energy electronic states near $E_{F}$. As a consequence of dynamical SWT we observe a transition from an insulating state to a metal. This prediction could be corroborated by future transport experiments on doped BP.

In Fig. 8 we show electrical and thermal transport properties of pure and electron/hole doped BP, calculated using bulk LDA+DMFT spectral functions of Fig. 7. The $T$ dependence of the thermoelectric power $S(T)$ in Fig. 8 shows fingerprints of doping-induced electronic modification across the semiconducting-to-metal transitions in doped BP. The fact that $S(T)$ changes sign from positive to negative around $150 \mathrm{~K}$ is a signature of the multiband character of transport linked to anisotropic reorganization of MO electronic states at low energies; see Fig. 7. Interestingly, the system spontaneously
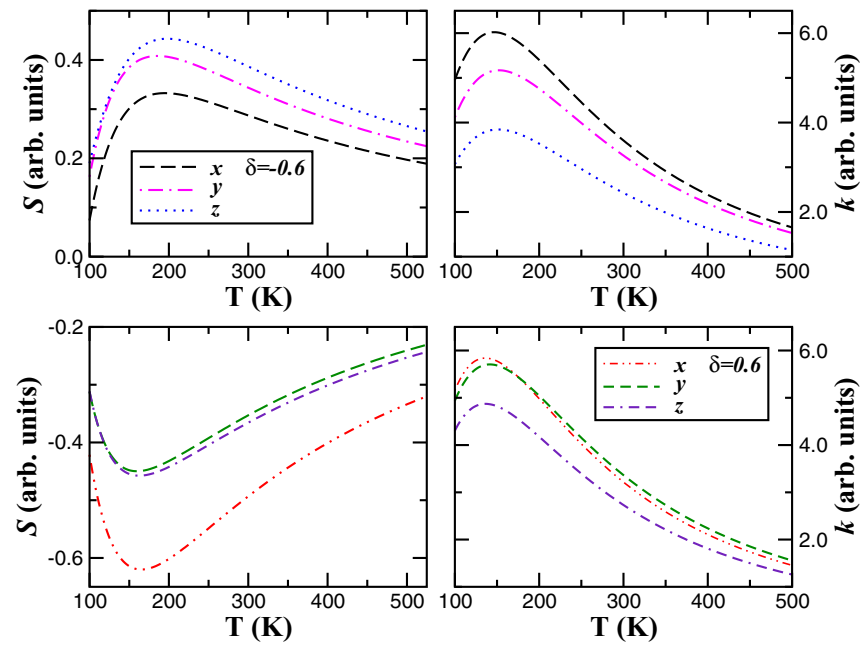

FIG. 9. Thermopower (left panels) and electronic thermal conductivity (right panels) of bulk BP along $x, y$, and $z$ directions as function of temperature at high doping levels, $\delta= \pm 0.6$, showing clear anisotropic thermal transport behavior upon electron/hole doping.

undergoes a charge reconstruction transition from a $p$ - to $n$-type material with doping: consistent with previous studies [3], negative $\delta$ corresponds to hole ( $p$-type) doping and gives positive $S$ above $200 \mathrm{~K}$, while positive $\delta$ corresponds to electron ( $n$-type) doping and provides negative Seebeck coefficient. In other words, in the $n$-type phase $S$ is negative with a minimum at $T=160 \mathrm{~K}$ for $\delta=0.6$, and in the $p$-type regime $S$ is positive with a broad maximum at temperatures above $180 \mathrm{~K}$. However, the fact that $S$ changes sign from positive to negative (or vice versa) around $100 \mathrm{~K}$ suggests dramatically different emergent charge carrier delocalization near the doping-induced semiconducting-to-metal transition in bulk BP.

The electronic carriers generated by doping and thermal activation of low-energy excitations have less pronounced effect in the electronic contribution to thermal conductivity, $k(T)$. As seen in Fig. 8, $k(T)$ above $150 \mathrm{~K}$ decreases significantly with increasing temperature in accordance with Ref. [4]. Below $100 \mathrm{~K} k(T)$ is clearly reduced compared to undoped BP, but the overall thermal conductivity is in semiqualitative agreement to values measured in Ref. [4]. Interestingly, $k(T)$ remains almost unaffected across the localization-delocalization transition at high temperatures, suggesting that its $T$ dependence is largely determined by a modest flow of thermal charge carriers due to Kondo-screened particle-hole excitations.

Moving to other transport properties, in the lower-right panels of Fig. 8 we display the $T$ dependence of the thermoelectric figure of merit, $Z T=\frac{T S^{2} \sigma_{d c}}{k}$, a measure of the thermoelectric efficiency of a system. As seen, at high temperatures $Z T$ is maximized upon electron/hole doping the parent semiconducting compound. Interestingly, below $100 \mathrm{~K}$ $Z T$ is strongly doping dependent, showing a sharp maximum around $25 \mathrm{~K}$. It is worth noting that similar behavior is found in $\mathrm{FeSb}_{2}$ [46], a narrow-gap semiconductor where it is attributed to strong electronic correlations effects [33]. Taken together, our results in Fig. 8 show the importance of electron/hole doping in maximizing the thermoelectric efficiency of bulk 
BP. Consistent with previous theoretical studies [3,32] our results demonstrate that $p-n$ doping might play an important role in tuning thermal transport properties of semiconducting materials with sizable electron-electron interactions.

While our results do not reproduce all features seen in extant thermal transport experiments of bulk BP [4], our estimation of the maximum value in $k(T)$ lies at somewhat higher $T$ than that found in bulk BP. However, the large anisotropy of $k(T)$ observed in single crystals with dominant $p$-type behavior [4] is well accounted for within LDA+DMFT for $U=10 \mathrm{eV}$ and $\delta=-0.6$; see top-right panel of Fig. 9. Although our results for the Seebeck coefficient show a distinct anisotropic trend compared to experiment, our results for $S(T)$ upon hole (n-type) doping (left-low panel of Fig. 9) are in good accord with theoretical estimations by Qin et al. [3]. The different behavior might be caused by a stronger tendency to localization in the $p$-type regime compared to $n$-type doping bulk BP. Hence, based on our theoretical prediction and on extant literature, we highlight the importance of $p-n$ doping and correlated electron physics for engineering novel strategies to improve the thermoelectric performance of bulk BP.

\section{CONCLUSION}

In conclusion, we have studied the role of localizationdelocalization transition on the electrical and thermal responses of bulk BP. The unusual anisotropic character of charge transport seen in experiments is interpreted in terms of orbital-selective, multiparticle electronic excitations. These arise from low-energy scatterings having their origin in the competition between selective-band localization and Kondo induced weak delocalization in LDA+DMFT. Good semi- quantitative agreement with polarized photoemission and inverse-photoemission spectra as well as electrical and thermal transport data puts our mechanism for bulk BP on solid ground. Based on our results we propose that doped BP and related layered hexagonal materials may have the potential to be of use as good thermoelectric materials. An attractive example to be mentioned here is $\mathrm{SnSe}$ [47], a thermoelectric material with similar puckered layer structure as bulk BP. Based on the fact that $\mathrm{SnSe}$ exhibits strong anisotropy of electron transport properties for both types of charge carriers [48], multiorbital Kondo correlation effects are expected to play an important role in tuning thermoelectric responses intrinsic to correlated semiconductors.

\section{ACKNOWLEDGMENTS}

L.C.'s work is supported by CNPq (Grant No. 307487/2014-8). Acknowledgment (L.C.) is also made to the Theoretical Chemistry department at Technical University Dresden for hospitality. S.L. acknowledges support from the UK Research Council for using work in the paper that was undertaken by a student under Project No. EP/M50631X/1 as well as the DFG for support under the priority project SPP 1415 and for a personal Heisenberg grant. S.L. also thanks ZIH Dresden and ARCCA Cardiff for computational resources. Via S.L.'s membership of the UK's HPC Materials Chemistry Consortium, which is funded by EPSRC (No. EP/L000202), this work made use of the facilities of ARCHER, the UK's National High-Performance Computing Service, which is funded by the Office of Science and Technology through EPSRC's High End Computing Programme. S.L. wishes to thank Min Gao, Cardiff for insightful discussions.
[1] A. Morita, Appl. Phys. A 39, 227 (1986); H. Liu, Y. Du, Y. Deng, and P. D. Ye, Chem. Soc. Rev. 44, 2732 (2015).

[2] F. Xia, H. Wang, and Y. Jia, Nat. Commun. 5, 4458 (2014).

[3] G. Qin, Q.-B. Yan, Z. Qin, S.-Y. Yue, H.-J. Cui, Q.-R. Zheng, and G. Su, Sci. Rep. 4, 6946 (2014).

[4] Y. Wang, G. Xu, Z. Hou, B. Yang, X. Zhang, E. Liu, X. Xi, Z. Liu, Z. Zeng, W. Wang, and G. Wu, Appl. Phys. Lett. 108, 092102 (2016).

[5] B. Aufray, A. Kara, S. Vizzini, H. Oughaddou, C. Léandri, B. Ealet, and G. L. Lay, Appl. Phys. Lett. 96, 183102 (2010); M. E. Dávila, L. Xian, S. Cahangirov, A. Rubio, and G. Le Lay, New J. Phys. 16, 095002 (2014).

[6] H. Liu, A. T. Neal, Z. Zhu, Z. Luo, X. Xu, D. Tománek, and P. D. Ye, ACS Nano 8, 4033 (2014); W. Lu, H. Nan, J. Hong, Y. Chen, C. Zhu, Z. Liang, X. Ma, Z. Ni, C. Jin, and Z. Zhang, Nano Res. 7, 853 (2014).

[7] S. Bagheri, N. Mansouri, and E. Aghaie, Int. J. Hydrogen Energy 41, 4085 (2016); L. M. Sandonas, D. Teich, R. Gutierrez, T. Lorenz, A. Pecchia, G. Seifert, and G. Cuniberti, J. Phys. Chem. C 120, 18841 (2016).

[8] C. Q. Han, M. Y. Yao, X. X. Bai, L. Miao, F. Zhu, D. D. Guan, S. Wang, C. L. Gao, C. Liu, D. Qian, Y. Liu, and J.-F. Jia, Phys. Rev. B 90, 085101 (2014).

[9] E. Golias, M. Krivenkov, and J. Sanchez-Barriga, Phys. Rev. B 93, 075207 (2016).
[10] Z. Hou, B. Yang, Y. Wang, B. Ding, X. Zhang, Y. Yao, E. Liu, X. Xi, G. Wu, Z. Zeng, Z. Liu, and W. Wang, Sci. Rep. 6, 23807 (2016).

[11] S. E. Boulfelfel, G. Seifert, Y. Grin, and S. Leoni, Phys. Rev. B 85, 014110 (2012).

[12] R. W. Keyes, Phys. Rev. 92, 580 (1953).

[13] Z. S. Popović, J. M. Kurdestany, and S. Satpathy, Phys. Rev. B 92, 035135 (2015).

[14] B. Kiraly, N. Hauptmann, A. N. Rudenko, M. I. Katsnelson, and A. A. Khajetoorians, Nano Lett. 17, 3607 (2017).

[15] Y. Hayasi, T. Takahashi, H. Asahina, T. Sagawa, A. Morita, and I. Shirotani, Phys. Rev. B 30, 1891 (1984).

[16] R. Schuster, J. Trinckauf, C. Habenicht, M. Knupfer, and B. Büchner, Phys. Rev. Lett. 115, 026404 (2015).

[17] Z. J. Xiang, G. J. Ye, C. Shang, B. Lei, N. Z. Wang, K. S. Yang, D. Y. Liu, F. B. Meng, X. G. Luo, L. J. Zou, Z. Sun, Y. Zhang, and X. H. Chen, Phys. Rev. Lett. 115, 186403 (2015).

[18] Y. Akahama, S. Endo, and S. Narita, Physica B+C 139-140, 397 (1986); see also K. Akiba, A. Miyake, Y. Akahama, K. Matsubayashi, Y. Uwatoko, and M. Tokunaga, Phys. Rev. B 95, 115126 (2017).

[19] J. Guo, H. Wang, F. von Rohr, W. Yi, Y. Zhou, Z. Wang, S. Cai, S. Zhang, X. Li, Y. Li, J. Liu, K. Yang, A. Li, S. Jiang, Q. Wu, T. Xiang, R. J. Cava, and L. Sun, arXiv:1611.03330.

[20] T. Kikegawa and H. Iwasaki, Acta Cryst. B 39, 158 (1983). 
[21] J. C. Jamieson, Science 139, 1291 (1963).

[22] P. W. Bridgman, J. Am. Chem. Soc. 36, 1344 (1914).

[23] R. Fei, V. Tran, and L. Yang, Phys. Rev. B 91, 195319 (2015).

[24] A. S. Rodin, A. Carvalho, and A. H. Castro Neto, Phys. Rev. Lett. 112, 176801 (2014)

[25] J. Kim, S. S. Baik, S. H. Ryu, Y. Sohn, S. Park, B.-G. Park, J. Denlinger, Y. Yi, H. J. Choi, and K. S. Kim, Science 349, 723 (2015).

[26] I. Shirotani, J. Mikami, T. Adachi, Y. Katayama, K. Tsuji, H. Kawamura, O. Shimomura, and T. Nakajima, Phys. Rev. B 50, 16274 (1994).

[27] M. Imada, A. Fujimori, and Y. Tokura, Rev. Mod. Phys. 70, 1039 (1998).

[28] L. Craco, M. S. Laad, S. Leoni, and A. S. de Arruda, Phys. Rev. B 87, 155109 (2013).

[29] L. Craco and S. Leoni, Sci. Rep. 5, 13772 (2015).

[30] G. Kotliar, S. Y. Savrasov, K. Haule, V. S. Oudovenko, O. Parcollet, and C. A. Marianetti, Rev. Mod. Phys. 78, 865 (2006).

[31] M. F. Pereira, Jr. and K. Henneberger, Phys. Status Solidi B 206, 477 (1998); D. S. Chemla and J. Shah, Nature (London) 411, 549 (2001).

[32] J. M. Tomczak, K. Haule, T. Miyake, A. Georges, and G. Kotliar, Phys. Rev. B 82, 085104 (2010).

[33] P. Sun, W. Xu, J. M. Tomczak, G. Kotliar, M. Sondergaard, B. B. Iversen, and F. Steglich, Phys. Rev. B 88, 245203 (2013).

[34] R. Martin and J. Allen, J. Appl. Phys. 50, 7561 (1979).

[35] M. Dzero, K. Sun, V. Galitski, and P. Coleman, Phys. Rev. Lett. 104, 106408 (2010)

[36] R. Wyckoff, Crystal Structures (Wiley, New York, 1963), Vol. 1.
[37] V. Antonov, B. Harmon, and A. Yaresko, Electronic Structure and Magneto-optical Properties of Solids (Kluwer Academic Publishers, Dordrecht, 2004). see also S. Chadov, X. Qi, J. Kübler, G. H. Fecher, C. Felser, and S. C. Zhang, Nat. Mater. 9 , 541 (2010).

[38] L. Craco, Phys. Rev. B 77, 125122 (2008).

[39] V. Y. Klevets, N. D. Savchenko, T. N. Shchurova, I. I. Opachko, and K. O. Popovic, Funct. Mater. 20, 97 (2013).

[40] D. A. Prishchenko, V. G. Mazurenko, M. I. Katsnelson, and A. N. Rudenko, 2D Mater. 4, 025064 (2017).

[41] C. Grenzebach, F. B. Anders, G. Czycholl, and T. Pruschke, Phys. Rev. B 74, 195119 (2006).

[42] L. Craco and M. S. Laad, Eur. Phys. J. B 89, 119 (2016).

[43] K. Urasaki and T. Saso, J. Phys. Soc. Jpn. 68, 3477 (1999); T. Saso, ibid. 73, 2894 (2004).

[44] L. Baldassarre, A. Perucchi, D. Nicoletti, A. Toschi, G. Sangiovanni, K. Held, M. Capone, M. Ortolani, L. Malavasi, M. Marsi, P. Metcalf, P. Postorino, and S. Lupi, Phys. Rev. B 77, 113107 (2008)

[45] J. F. DiTusa, K. Friemelt, E. Bucher, G. Aeppli, and A. P. Ramirez, Phys. Rev. B 58, 10288 (1998); see also M. S. Laad, L. Craco, and E. Müller-Hartmann, ibid. 67, 033105 (2003).

[46] P. Sun, N. Oeschler, S. Johnsen, B. B Iversen, and F. Steglich, Phys. Rev. B 79, 153308 (2009).

[47] L.-D. Zhao, S.-H. Lo, Y. Zhang, H. Sun, G. Tan, C. Uher, C. Wolverton, V. P. Dravid, and M. G. Kanatzidis, Nature (London) 508, 373 (2014); L.-D. Zhao, G. Tan, S. Hao, J. He, Y. Pei, H. Chi, H. Wang, S. Gong, H. Xu, V. P. Dravid, C. Uher, G. J. Snyder, C. Wolverton, and M. G. Kanatzidis, Science 351, 141 (2016).

[48] K. Kutorasinski, B. Wiendlocha, S. Kaprzyk, and J. Tobola, Phys. Rev. B 91, 205201 (2015). 